\title{
Avaliação das opiniões dos graduandos na área da saúde frente a pessoas portadoras de transtornos mentais
}

\author{
Evaluation of the opinion of undergraduate students in the health area regardling people with \\ mental disorders
}

Evaluación de las opiniones de los estudiantes de pregrado del área de la salud sobre personas con trastornos mentales

Recebido: 22/10/2021 | Revisado: 28/10/2021 | Aceito: 29/10/2021 | Publicado: 01/11/2021

Letícia Souza Carvalho

ORCID: https://orcid.org/0000-0002-1849-6345 Universidade Federal de Sergipe, Brasil E-mail: leticia.socarvalho11@gmail.com Isis Grabrielly Lima Santana ORCID: https://orcid.org/0000-0002-2575-7312 Universidade Federal de Sergipe, Brasil E-mail: isis_gabrielly123@hotmail.com

Carla Kalline Alves Cartaxo Freitas ORCID: https://orcid.org/0000-0001-7604-9132 Universidade Federal de Sergipe, Brasil E-mail: carlakalline@gmail.com Arthur de Almeida Medeiros ORCID: https://orcid.org/0000-0002-2192-8823 Universidade Federal de Mato Grosso do Sul, Brasil E-mail: aamedeiros.ufms@gmail.com

Alysson de Jesus Santos

ORCID: https://orcid.org/0000-0003-0202-341X Universidade Federal de Sergipe, Brasil E-mail: alyssonjst@gmail.com

Bárbara Giovanna de Araújo Santos ORCID: https://orcid.org/0000-0003-2499-6634 Universidade Federal de Sergipe, Brasil E-mail: babi.k3.love@gmail.com

Maria do Socorro Claudino Barreiro

ORCID: https://orcid.org/0000-0001-9823-4638 Universidade Federal de Sergipe, Brasil E-mail: socorrocbarreiro@gmail.com

Matheus Santos Melo

ORCID: https://orcid.org/0000-0002-9151-8467 Universidade Federal de Sergipe, Brasil

E-mail: matheussmelo@live.com

Thaiane Santana Santos

ORCID: https://orcid.org/0000-0003-2207-8055 Universidade Federal de Sergipe, Brasil E-mail: thaianesantana08@gmail.com

Glebson Moura Silva

ORCID: https://orcid.org/0000-0002-4977-2787 Universidade Federal de Sergipe, Brasil E-mail: glebsonmoura@yahoo.com.br

\begin{abstract}
Resumo
Objetivo: Avaliar a opinião e atitudes dos estudantes universitários da área da saúde frente às pessoas com transtornos mentais. Materiais e métodos: Pesquisa quantitativa e transversal, com abordagem descritiva, realizada com 328 discentes dos cursos de graduação de uma Universidade Federal do nordeste brasileiro. Foram utilizadas a Escala de Opiniões sobre Doença Mental e um questionário de caracterização do perfil sociodemográfico dos participantes. Ademais, para análise dos dados foram utilizados os testes de Kolmogorov-Smirnov, Mann-Whitney e Kruskal-Wallis. Resultados: O estudo constatou que Medicina foi o curso responsável pelo menor nível de estigma e Odontologia, o de maior estigma. Percebeu-se que os discentes do primeiro ano possuíam maior grau de estigma, em relação aos alunos dos últimos anos da graduação. Tal dado tamnbém foi prevalente entre indivíduos do sexo masculino. Pôde-se observar diferença nas vertentes de sexo, curso, ano de graduação, a disciplina de saúde mental e o contato com pessoas com
\end{abstract}


transtornos mentais. Já nos domínios, obteve-se significância estatística em todos, não obstante que cada vertente correlacionou-se com um grupo diferente de categorias. Conclusões: O estigma é uma das principais barreiras no cuidado às pessoas com transtornos mentais. Sendo assim, a associação de conteúdos teóricos com a prática em estágios durante a graduação é a principal forma de reduzi-lo.

Palavras-chave: Estigma social; Transtornos mentais; Estudantes; Educação superior.

\begin{abstract}
Objective: To evaluate the opinion and attitudes of university students in the health area towards people with mental disorders. Materials and methods: Quantitative and cross-sectional research, with a descriptive approach, carried out with 328 undergraduate students at a Federal University in northeastern Brazil. The Opinions Scale on Mental Illness and a questionnaire to characterize the sociodemographic profile of the participants were used. Furthermore, for data analysis, the Kolmogorov-Smirnov, Mann-Whitney and Kruskal-Wallis tests were used. Results: The study found that Medicine was the course responsible for the lowest level of stigma and Dentistry, the one with the highest stigma. It was noticed that first-year students had a higher degree of stigma compared to students in the last years of graduation. This data was also prevalent among male individuals. Differences were observed in terms of gender, course, year of graduation, mental health discipline and contact with people with mental disorders. In the domains, statistical significance was obtained in all, although each strand correlated with a different group of categories. Conclusions: Stigma is one of the main barriers in caring for people with mental disorders. Thus, the association of theoretical content with internship practice during graduation is the main way to reduce it.
\end{abstract}

Keywords: Social stigma; Mental disorders; Students; College education.

\begin{abstract}
Resumen
Objetivo: Evaluar la opinión y actitudes de los estudiantes universitarios del área de la salud hacia las personas con trastornos mentales. Materiales y métodos: Investigación cuantitativa y transversal, con enfoque descriptivo, realizada con 328 estudiantes de pregrado de una Universidad Federal en el noreste de Brasil. Se utilizó la Escala de Opinión sobre Enfermedad Mental y un cuestionario para caracterizar el perfil sociodemográfico de los participantes. Además, para el análisis de los datos se utilizaron las pruebas de Kolmogorov-Smirnov, Mann-Whitney y Kruskal-Wallis. Resultados: El estudio encontró que Medicina fue el curso responsable del menor nivel de estigma y Odontología, el de mayor estigma. Se notó que los estudiantes de primer año tenían un mayor grado de estigma en comparación con los estudiantes de los últimos años de graduación. Estos datos también prevalecieron entre los hombres. Se observaron diferencias en cuanto a género, curso, año de graduación, disciplina de salud mental y contacto con personas con trastornos mentales. En los dominios, se obtuvo significación estadística en todos, aunque cada capítulo se correlacionó con un grupo diferente de categorías. Conclusiones: El estigma es una de las principales barreras en el cuidado de personas con trastornos mentales. Así, la asociación del contenido teórico con la práctica de prácticas durante la graduación es la principal vía para reducirlo.
\end{abstract}

Palabras clave: Estigma social; Desordenes mentales; Estudiantes; Educación universitaria.

\title{
1. Introdução
}

Os transtornos mentais são muito abrangentes e estão relacionados à redução da capacidade psíquica, aos transtornos de personalidade e à dependência de substâncias, como álcool e outras drogas (Organização Mundial da Saúde [OMS], 2005). Outras características são que eles afetam tanto a saúde pessoal quanto a familiar, além de serem responsáveis pela morte precoce dos portadores e por grande parte dos gastos em doenças não notificáveis (Rocha, Hara, \& Paprocki, 2015).

Os indivíduos com transtorno metal foram tratados, historicamente, como loucos, perigosos, doentes, anormais, havendo distintas concepções sobre a temática e, consequentemente, pensamentos ambíguos (Cândido et al., 2012). Isso configura-se justamente ao estigma, sendo que essas pessoas são negligenciadas e discriminadas, acarretando em sofrimento, limitação, medo e preconceito a si mesmo (Silva, \& Carvalho, 2017; Thornicroft et al., 2016).

Nesse contexto, infere-se que o estigma se correlaciona ao estado de pré-julgamento, pois ele não está associado apenas a características vistas e reais, englobando sinais e comportamentos invisíveis, imaginados e criados pelas pessoas, julgando os indivíduos que tem transtorno mental antes mesmo de conhecê-los (Ferreira, \& Carvalho, 2017).

A vivência das consequências do estigma é algo corriqueiro ao longo da história, e faz as pessoas com transtorno psicológico se isolarem no intuíto de preservar a sua identidade, esquivar-se da exclusão social e do desprezo, e evitar o agravo das sensações por elas sentidas (Jorge, 2013). A família destas pessoas também sofrem com a vergonha, o remorso e a preocupação, além de possuir problemas emocionais e econômicos. Há, ao mesmo tempo, um déficit de apoio familiar que gera 
a exacerbação do estresse (Rocha et al., 2015).

A existência de atitudes e opiniões negativas na sociedade em geral, inclusive entre os estudantes e os profissionais da saúde, faz com que as pessoas em sofrimento psíquico sejam consideradas diferentes dos outros indivíduos, e, consequentemente, há uma diferenciação no cuidado à saúde, atingindo negativamente o tratamento geral e específico do transtorno mental (Ordan, Shor, Liebergall-Wischnitzer, Noble, \& Noble, 2018; Vistore et al., 2018).

As consequências negativas do estigma dentre os estudantes e profissionais da saúde englobam o atraso na busca por cuidados, de modo que essa aconteça apenas em estágios avançados da afecção, gerando dificuldade no diagnóstico, no tratamento e na recuperação, além de comprometer o serviço de saúde de forma que haja redução nos investimentos e, consequentemente, da qualidade do atendimento (Mathew, 2017).

Uma das estratégias para modelar as opiniões e atitudes de forma a reduzir o estigma é a educação, pois o ambiente acadêmico exerce um papel importante na formação das atitudes dos estudantes em relação às pessoas com transtornos mentais, já que a falta de consciência e compreensão predispõe a estigmatização (Corrigan, Morris, Michaels, Rafacz, \& Rüsch, 2012).

Essa mitagação do estigma é alcançada por meio da modificação das falsas crenças e desinformações que englobam o transtorno mental, o que irá melhorar quali e quantitativamente os conhecimentos sobre essa temática (Arboleda-Flórez, \& Stuart, 2012). Dessa forma, é esperado que haja amenização das opiniões e atitudes negativas dos estudantes da área da saúde ao longo da graduação, pois nesse período eles irão adquirir muitas informações acerca da saúde mental, o que irá colaborar para com esse resultado.

Nesse contexto, o presente estudo visa avaliar a opinião dos estudantes universitários da área da saúde frente às pessoas com transtornos mentais, já que a presença de estigma poderá refletir na prestação de uma assistência de qualidade.

\section{Metodologia}

Trata-se de uma pesquisa transversal e quantitativa, com abordagem descritiva e realizada numa Universidade Federal do nordeste brasileiro estruturada por oito cursos de graduação na área de saúde: Enfermagem, Nutrição, Fonoaudiologia, Medicina, Terapia Ocupacional, Odontologia, Fisioterapia e Farmácia. Essa instituição utiliza metodologias ativas de ensino e seus projetos pedagógicos estão estruturados a partir da Aprendizagem Baseada em Problemas (ABP), uma metodologia de ensino-aprendizagem ativa motivada pela resolução de problemas (Silva, Vilaça, Martins, \& Augusto, 2015), além de outras estratégias de ensino.

Foram incluídos na pesquisa os acadêmicos do primeiro e último ano dos oito cursos ofertados no Campus, maiores de 18 anos. Adotou-se como critério de exclusão o preenchimento incompleto ou incorreto das escalas.

A pesquisa foi realizada entre setembro de 2019 e julho de 2020 com o auxílio de uma equipe de quatro pesquisadores que receberam treinamento prévio para aplicação dos questionários e escalas selecionados para o estudo. O contato e a abordagem com os graduandos foram feitos de forma presencial. Apenas 23 alunos contatados não aceitaram participar da pesquisa. Dessa forma, a amostra da pesquisa foi composta por 328 alunos.

O instrumento utilizado foi a Escala de Opiniões sobre Doença Mental (ODM) de Cohen e Struening, versão traduzida e validada para o Brasil (Rodrigues, 1983), sendo que também foi avaliado o perfil sociodemográfico dos participantes através de um questionário elaborado pelos pesquisadores. A Escala ODM é composta de 51 afirmações do tipo likert, agrupadas em 7 dimensões com os seguintes conceitos (Delevati, \& Palazzo, 2008):

-Autoritarismo (questões 21, 26, 31, 32, 34, 36, 40, 45 e 49): reflete a perspectiva de que o portador de transtorno mental necessita ser isolado de outros pacientes, permanecendo sob portas trancadas e vigilância. Contém o conceito da irrecuperabilidade pessoal e social do indivíduo e a ideia de sua periculosidade.

-Benevolência (questões 12,18,22, 23 e 38): traduz a visão de que portador de transtorno mental, devido à sua 
infelicidade, deve ser amparado por meio de protecionismo bondoso e paternalista, com base em cuidados, atenção pessoal e conforto material.

-Ideologia da higiene mental (questões 2, 3, 8, 13, 27, 28, 33, 41, 44, 47 e 50): representa a ideia de que o portador de transtorno mental é uma pessoa semelhante às pessoas normais, com diferenças quantitativas, porém, não qualitativas. Podem desempenhar atividades especializadas e até cuidar de crianças.

-Restrição social (questões 4, 14, 19, 24, 37, 39 e 51): traduz o transtorno mental como uma espécie de defeito hereditário, completamente diferente de outras doenças, cujo portador pode contaminar a família e a sociedade, devendo, portanto, ser protegida por meio de restrição dos direitos pessoais e sociais do paciente, mesmo após a hospitalização.

-Etiologia interpessoal (questões 5, 10, 20, 25, 30 e 35): explica transtorno mental como originário de vivências interpessoais, com ênfase para a interação com figuras parentais.

-Etiologia de esforço mental (questões 1, 7, 9, 15, 16, 43, 46 e 48): reflete a ideia de que o transtorno mental se origina de "excessivo esforço cerebral" pelo trabalho intelectual exagerado, por pensar demais ou por ter pensamentos negativos.

-Visão minoritária (questões 6, 11, 17, 29 e 42): traduz o conceito de que portador de transtorno mental, por ser muito diferente das pessoas consideradas normais, pode ser facilmente reconhecido em um agrupamento humano, principalmente pela sua aparência externa.

Nesta escala, os resultados baixos em cada dimensão correspondem a níveis superiores de atitudes estigmatizantes, à exceção do fator Ideologia de Higiene Mental, o qual os níveis altos de atitudes negativas resultam de escores elevados (Rodrigues, 1983).

Os dados foram tabulados no software IBM® SPSS - Statistical Package for the Social Sciences 23.0 Mac (SPSS 20.0 Mac, SPSS Inc., Chicago, Illinois, EUA). Procedeu-se análise descritiva dos dados com apresentação dos resultados relativos as variáveis categóricas em frequência absoluta e relativa, e para as variáveis quantitativas em média, desvio padrão, mediana e valores mínimos e máximos. Após a verificação da normalidade dos dados através do teste de Kolmogorov-Smirnov, utilizado para medir a distância entre os resultados de uma distribuição a ser testada e os valores associados à distribuição verdadeira hipoteticamente (Araújo, Sousa, Ribeiro, Santos, \& Medeiros, 2008), foram realizados os testes de Mann-Whitney para as variáveis independentes dicotômicas (Perme, \& Manevski, 2019) e de Kruskal-Wallis para as variáveis independentes policotômicas (McKight, \& Najab, 2010).

Nos casos em que houveram diferenças significativas identificadas no teste de Kruskal-Wallis procederam-se os testes par-a-par com correção de Bonferroni para identificar entre quais grupos estavam presentes tais diferenças (Weisstein, 2004). Todas as análises foram realizadas no software SPSS 25.0 e foi considerado nível de significância de 5\%.

Ademais, os escores fatoriais brutos obtidos foram transformados para o sistema Sten, que proporciona uma comparação padrão entre os fatores, variando entre 1 e 10, $(M=5,5 ; D P=0,5)$ (Canfield, 1951), o que significa que as respostas esperadas ficam entre os valores de 5 e 6.

Com o intuito de assegurar e eficácia dos aspectos éticos, o presente estudo respeitou os preceitos da Resolução de Pesquisa envolvendo Seres Humanos nº 466/2012, do Conselho Nacional de Ética em Pesquisa (CONEP) (Brasil, 2012).

O estudo foi enviado ao Comitê de Ética em Pesquisa (CEP) da Universidade Federal de Sergipe e aprovado com o Parecer $\mathrm{n}^{\circ}$ 3.573.141. Todos os participantes envolvidos na pesquisa foram informados dos objetivos, e, após a leitura e explicações apresentadas, concordaram e assinaram o Termo de Consentimento Livre e Esclarecido (TCLE).

\section{Resultados}

A amostra foi constituída por um total de 328 estudantes com média de 22,66 anos de idade, sendo 171 (52,1\%) do primeiro ano e 157 (47,9\%) do último ano dos oito cursos da área da saúde: Enfermagem (12,8\%), Medicina (13,7\%), Farmácia 
(9,5\%), Terapia Ocupacional (15,2\%), Nutrição (10,4\%), Odontologia (12,5\%), Fisioterapia (19,5\%) e Fonoaudiologia (6,4\%). Desses, 75,6\% afirmaram que não cursaram disciplina de saúde mental durante sua formação e 23,8\% afirmaram ter cursado. Em relação ao tipo de aula, 19,2\% responderam aulas teóricas e práticas enquanto 4,6\% apenas teóricas.

A maioria afirma que têm contato com pessoas que possuem transtorno mental $(57,0 \%)$, representados por familiares $(58,8 \%)$ e mantêm uma frequência de contato ocasional com eles $(33,1 \%)$, além disso relatam não apresentar doenças físicas ou mentais $(84,8 \%)$. Para aqueles que possuem alguma patologia, 50\% foi mental e 50\% física e 8,2\% utilizam serviço de saúde mental. As informações até então mencionadas estão representadas na Tabela 1.

Tabela 1: Análise descritiva das variáveis quantitativas incluídas no estudo relacionadas aos estudantes da Universidade Federal de Sergipe. Lagarto/ Sergipe, 2020.

\begin{tabular}{|c|c|c|}
\hline Variáve & & \\
\hline \multirow[t]{2}{*}{ Idade } & Média & $\mathbf{D P}$ \\
\hline & 22,66 & 4,67 \\
\hline Sexo & $\mathbf{n}$ & $\%$ \\
\hline Feminino & 222 & 67,7 \\
\hline Masculino & 106 & 32,3 \\
\hline \multicolumn{3}{|l|}{ Curso } \\
\hline Enfermagem & 45 & 13,7 \\
\hline Farmácia & 31 & 9,5 \\
\hline Terapia Ocupacional & 50 & 15,2 \\
\hline Nutrição & 34 & 10,4 \\
\hline Odontologia & 41 & 12,5 \\
\hline Fisioterapia & 64 & 19,5 \\
\hline Fonoaudiologia & 21 & 6,4 \\
\hline \multicolumn{3}{|l|}{ Ciclo da graduação } \\
\hline Primeiro & 171 & 52,1 \\
\hline Último & 157 & 47,9 \\
\hline \multicolumn{3}{|c|}{ Disciplina sobre saúde mental durante a formação } \\
\hline Sim & 78 & 23,8 \\
\hline Não & 248 & 75,6 \\
\hline Sistema & 2 & 0,6 \\
\hline \multicolumn{3}{|c|}{ Contato com pessoas que possuem transtorno mental } \\
\hline Sim & 187 & 57 \\
\hline Não & 139 & 42,4 \\
\hline Não respondeu & 2 & 0,6 \\
\hline
\end{tabular}

Legenda: n= Frequência absoluta, \%= Frequência relativa. Fonte: Autores.

As médias, medianas e desvio padrão para os escores dos fatores da escala ODM foram exibidos na Tabela 2. A pontuação dos domínios do instrumento apresentaram escores muito próximos entre si com pouca variação em todos os domínios, com valores oscilando entre 5,49 e 5,51. Destarte, pode-se inferir que houve presença de opiniões negativas nos dados coletados. 
Tabela 2: Análise descritiva das variáveis quantitativas da Escala ODM relacionadas aos estudantes da Universidade Federal de Sergipe. Lagarto/Sergipe, 2020.

\begin{tabular}{lccccc}
\hline \multicolumn{1}{c}{ Variáveis } & Média & DP & Mediana & Mínimo & Máximo \\
\hline Autoritarismo & 5,51 & 0,49 & 5,59 & 3 & 6 \\
Benevolência & 5,5 & 0,5 & 5,42 & 5 & 8 \\
Ideologia da Higiene Mental & 5,49 & 0,48 & 5,46 & 4 & 7 \\
Restrição Social & 5,51 & 0,5 & 5,58 & 3 & 7 \\
Etiologia Interpessoal & 5,5 & 0,5 & 5,52 & 4 & 7 \\
Etiologia de Esforço Mental & 5,51 & 0,5 & 5,53 & 4 & 6 \\
Visão Minoritária & 5,51 & 0,49 & 5,5 & 4 & 7 \\
\hline
\end{tabular}

Legenda: DP= Desvio Padrão. Fonte: Autores.

É válido salientar que, embora existam poucas variações, devido às proximidades entre as médias, houve significância estatística em alguns aspectos. As variáveis que obtiveram diferença significativa foram: sexo, curso, ciclo de graduação, presença de disciplina de saúde mental durante a graduação e contato com pessoas com transtorno mental (Tabela 3).

No que concerne à variável sexo, os domínios autoritarismo $(\mathrm{p}=0,028)$, benevolência $(\mathrm{p}=0,001)$, restrição social ( $\mathrm{p}=0,016)$, etiologia interpessoal $(\mathrm{p}=0,018)$, etiologia de esforço mental $(\mathrm{p}=0,004)$ e visão minoritária $(\mathrm{p}=0,034)$ apresentaram diferença significativa, sendo que houve prevalência de médias menores no sexo masculino em todas dimensões, exceto na de benevolência, indicando que este possui maior estigma em relação ao sexo feminino.

Tabela 3: Média dos escores significativos estatisticamente dos domínios da escala ODM de acordo com as variáveis independentes. Lagarto/Sergipe, 2020.

\begin{tabular}{|c|c|c|c|c|c|c|c|}
\hline \multirow[b]{2}{*}{ Variáveis } & \multirow[b]{2}{*}{$\begin{array}{c}\text { Ajuda } \\
\text { Média } \pm D P\end{array}$} & \multicolumn{3}{|c|}{ Ideologia de } & \multicolumn{3}{|c|}{ Etiologia } \\
\hline & & $\begin{array}{c}\text { Benevolência } \\
\text { Média } \pm D P\end{array}$ & $\begin{array}{c}\text { Higiene } \\
\text { Mental } \\
\text { Média } \pm D P\end{array}$ & $\begin{array}{c}\text { Restrição } \\
\text { Social } \\
\text { Média } \pm D P\end{array}$ & $\begin{array}{c}\text { Etiologia } \\
\text { Interpessoal } \\
\text { Média } \pm D P\end{array}$ & $\begin{array}{c}\text { Esforço } \\
\text { Mental } \\
\text { Média } \pm D P\end{array}$ & $\begin{array}{c}\text { Visão } \\
\text { Minoritária } \\
\text { Média } \pm D P\end{array}$ \\
\hline \multicolumn{8}{|l|}{ Sexo } \\
\hline Feminino & $5,57 \pm 0,44$ & $5,44 \pm 0,48$ & $5,45 \pm 0,47$ & $5,56 \pm 0,46$ & $5,53 \pm 0,46$ & $5,57 \pm 0,46$ & $5,56 \pm 0,47$ \\
\hline Masculino & $5,41 \pm 0,56$ & $5,61 \pm 0,52$ & $5,52 \pm 0,48$ & $5,41 \pm 0,54$ & $5,41 \pm 0,51$ & $5,39 \pm 0,53$ & $5,43 \pm 0,48$ \\
\hline $\mathrm{p}_{\dagger}^{\dagger}$ & $0,028 *$ & $0,001 *$ & 0,214 & $0,016 *$ & $0,018 *$ & $0,004 *$ & $0,034 *$ \\
\hline \multicolumn{8}{|l|}{ Curso } \\
\hline Enfermagem & $5,51 \pm 0,49 a b$ & $5,58 \pm 0,51$ & $5,49 \pm 0,48$ & $5,45 \pm 0,47$ & $5,50 \pm 0,44$ & $5,47 \pm 0,40$ & $5,51 \pm 0,47$ \\
\hline Medicina & $5,65 \pm 0,55 \mathrm{a}$ & $5,50 \pm 0,49$ & $5,38 \pm 0,44$ & $5,66 \pm 0,54$ & $5,58 \pm 0,55$ & $5,65 \pm 0,54$ & $5,64 \pm 0,55$ \\
\hline Farmácia & $5,50 \pm 0,51 \mathrm{ab}$ & $5,31 \pm 0,29$ & $5,46 \pm 0,47$ & $5,41 \pm 0,52$ & $5,43 \pm 0,49$ & $5,41 \pm 0,45$ & $5,51 \pm 0,45$ \\
\hline \multicolumn{8}{|l|}{ Terapia } \\
\hline Ocupacional & $5,62 \pm 0,37 \mathrm{ab}$ & $5,44 \pm 0,48$ & $5,43 \pm 0,45$ & $5,60 \pm 0,38$ & $5,50 \pm 0,47$ & $5,50 \pm 0,51$ & $5,50 \pm 0,42$ \\
\hline Nutrição & $5,62 \pm 0,37 \mathrm{ab}$ & $5,44 \pm 0,48$ & $5,43 \pm 0,45$ & $5,60 \pm 0,38$ & $5,50 \pm 0,47$ & $5,50 \pm 0,51$ & $5,50 \pm 0,42$ \\
\hline Odontologia & $5,29 \pm 0,59 b$ & $5,71 \pm 0,68$ & $5,59 \pm 0,53$ & $5,38 \pm 0,56$ & $5,48 \pm 0,50$ & $5,40 \pm 0,62$ & $5,40 \pm 0,51$ \\
\hline Fisioterapia & $5,52 \pm 0,50 \mathrm{ab}$ & $5,42 \pm 0,48$ & $5,42 \pm 0,50$ & $5,52 \pm 0,55$ & $5,49 \pm 0,49$ & $5,58 \pm 0,47$ & $5,56 \pm 0,47$ \\
\hline
\end{tabular}




$\begin{array}{lccccccc}\text { Fonoaudiologia } & 5,37 \pm 0,27 \mathrm{~b} & 5,40 \pm 0,30 & 5,65 \pm 0,48 & 5,50 \pm 0,34 & 5,39 \pm 0,28 & 5,49 \pm 0,39 & 5,43 \pm 0,34 \\ \mathrm{p} \dagger \dagger & 0,002^{*} & 0,044^{*} & 0,346 & 0,134 & 0,609 & 0,152 & 0,518\end{array}$

\section{Ciclo da graduação}

$\begin{array}{lccccccc}\text { Primeiro } & 5,45 \pm 0,48 & 5,49 \pm 0,53 & 5,50 \pm 0,47 & 5,45 \pm 0,47 & 5,39 \pm 0,48 & 5,45 \pm 0,49 & 5,42 \pm 0,45 \\ \text { Último } & 5,59 \pm 0,47 & 5,50 \pm 0,46 & 5,45 \pm 0,48 & 5,59 \pm 0,50 & 5,61 \pm 0,45 & 5,57 \pm 0,48 & 5,63 \pm 0,48 \\ \text { p } \dagger & 0,001 * & 0,51 & 0,423 & 0,002^{*} & <0,001^{*} & 0,010^{*} & <0,001^{*}\end{array}$

Disciplina sobre saúde mental durante a formação

$\begin{array}{lccccccc}\text { Sim } & 5,65 \pm 0,50 & 5,44 \pm 0,45 & 5,40 \pm 0,45 & 5,64 \pm 0,52 & 5,58 \pm 0,48 & 5,59 \pm 0,49 & 5,61 \pm 0,50 \\ \text { Não } & 5,48 \pm 0,47 & 5,51 \pm 0,51 & 5,49 \pm 0,48 & 5,48 \pm 0,48 & 5,47 \pm 0,48 & 5,48 \pm 0,49 & 5,49 \pm 0,47 \\ \mathrm{p} \dagger & <0,001 * & 0,315 & 0,167 & 0,002^{*} & 0,044^{*} & 0,042^{*} & 0,046^{*}\end{array}$

\section{Contato com pessoas que possuem transtorno mental}

\begin{tabular}{lccccccc} 
Sim & $5,55 \pm 0,49$ & $5,47 \pm 0,48$ & $5,43 \pm 0,50$ & $5,57 \pm 0,51$ & $5,52 \pm 0,48$ & $5,55 \pm 0,49$ & $5,59 \pm 0,50$ \\
Não & $5,47 \pm 0,47$ & $5,52 \pm 0,52$ & $5,52 \pm 0,44$ & $5,44 \pm 0,46$ & $5,45 \pm 0,48$ & $5,45 \pm 0,48$ & $5,42 \pm 0,43$ \\
$\mathrm{p} \dagger$ & $0,033^{*}$ & 0,42 & $0,048 *$ & $0,004^{*}$ & 0,162 & $0,038^{*}$ & $0,001^{*}$ \\
\hline
\end{tabular}

Legenda: DP: Desvio Padrão; Teste de Mann-Whitney; ${ }^{\dagger T}$ Teste de Kruskall-Wallis; *Estatisticamente Significativo a nível de $5 \%$. Fonte: Autores.

Referente aos cursos, constatou-se atores significativos nas categorias autoritarismo $(p=0,002)$ e benevolência $(\mathrm{p}=0,044)$, porém somente $\mathrm{o}$ autoritarismo teve diferença identificada entre os estudantes do curso de Medicina que obtiveram menor estigmatização em relação aos de Odontologia e Fonoaudiologia que evidenciaram maiores atitudes estigmatizantes.

Sobre o ciclo de graduação, os domínios significativos foram o autoritarismo $(\mathrm{p}=0,001)$, a restrição social $(\mathrm{p}=0,002)$, a etiologia interpessoal $(\mathrm{p}<0,001)$, a etiologia de esforço mental $(\mathrm{p}=0,010)$ e a visão minoritária $(\mathrm{p}<0,001)$. Diante disso, percebeu que os estudantes do primeiro ciclo apresentaram menores médias e, consequentemente, maior estigma em comparação aos do último ciclo.

Na variável disciplina sobre saúde mental, os domínios estatisticamente significativos foram o autoritarismo ( $<<0,001)$, restrição social ( $\mathrm{p}=0,002)$, etiologia interpessoal $(\mathrm{p}=0,044)$, etiologia de esforço mental $(\mathrm{p}=0,042)$ e visão minoritária $(\mathrm{p}=0,046)$. Nesse contexto, os universitários que tiveram esse módulo curricular apresentaram reduzidas atitudes estigmatizantes em detrimento àqueles que não o tiveram.

No que se refere ao contato com pessoas com transtorno mental, foi evidenciado que acadêmicos com essa relação apresentam menor estigmatização comparado com os que não possuem. Além disso, foram considerados significativos os domínios autoritarismo $(p=0,033)$, ideologia de higiene mental $(p=0,048)$, restrição social $(p=0,004)$, etiologia de esforço mental $(\mathrm{p}=0,038)$ e visão minoritária $(\mathrm{p}=0,001)$.

\section{Discussão}

$\mathrm{Na}$ análise da ODM, foi observado que o sexo masculino apresentou maior estigma na maioria das dimensões. Esse achado foi congruente com uma pesquisa realizada com estudantes de Medicina e Enfermagem na Índia, na qual as mulheres tiveram atitudes menos restritivas e mais benevolentes e isso pode ser justificado pelo fato que o sexo masculino geralmente possui características mais restritivas e limitadas quando comparado às mulheres que são mais propensas a praticar o acolhimento devido à ligação de traços maternos (Poreddi, Thimmaiah, \& Math, 2017).

Diante dessa divergencia, outro fator que pode ter contribuido para ese resultado é que a maioria dos partipantes foram do sexo feminino, representando uma amostra maior do que o dobro dos homens integrantes, o que provoca uma condensação 
da visão menos estigmatizante que as mulheres possuem.

Nessa pesquisa, Terapia Ocupacional, Nutrição e Medicina destacaram-se na redução de atitudes estigmatizantes relacionadas ao autoritarismo e os cursos Odontologia, Enfermagem e Medicina, à benevolência. Essa amenização no estigma pode estar correlacionada com a forma de aprendizado e com a metodologia adotada na instituição de ensino. Há literaturas que fundamentam essa hipótese ao abordar três vertentes relacionada com a metodologia de instrução acadêmica, são elas: a influência positiva, os efeitos negativos e a permanência de atitudes negativas (Lim et al., 2019; Ferreira et al., 2015; Sari, \& Yuliastuti, 2018).

Em contrapartida, outro estudo (Ferreira, \& Carvalho, 2020), que teve como população 38 alunos de enfermagem, demonstrou que eles possuem atitudes mais positivas no domínio autoritarismo, inferindo um resultado distinto quando comparado a esta pesquisa.

O fato do curso Medicina obter taxas reduzidas de estigma em ambas categorias supracitadas no presente estudo direciona à possibilidade de um atendimento mais humanizado, que denote maior liberdade e protecionismo às pessoas em sofrimento mental. Porém, há estudo que contrapõe esse resultado ao afirmar que esta formação prioriza o tratamento farmacológico, além de possuir características autoritárias e hierarquizadas (Barbosa, 2010).

Verificou-se ainda que os discentes de Fonoaudiologia mostraram níveis maiores de atitudes estigmatizantes nas dimensões de autoritarismo e benevolência. Pode-se explicar esse resultado pelo fato de que esses cursos não têm contato precoce com pessoas portadoras de transtornos mentais o que acarreta em acentuação do estigma nessa população. Outra hipótese que pode ser levantada é devido à falta de um módulo específico sobre saúde mental na estrutura curricular desses cursos na amostra estudada (Resolução CONEP n. 22, 2015; Resolução CONEP n. 25, 2017).

A ausência de contato precoce com o transtorno mental durante a formação pode alavancar prejuízos na adaptação e nos conhecimentos acerca dos cuidados necessários nessa condição. Ademais, essa acentuação nos níveis de estigma também pode ser relativa à falta de interesse em atuar na área de saúde mental, além da composição dos componentes teóricos e da forma de treinamento durante a graduação (Fresán, Robles-García, Martínez-López, Tovilla-Zárate, \& Madrigal, 2018; Chang et al., 2017; Aruna et al., 2016).

Esse resultado também está correlacionado com outras duas variáveis da pesquisa: ciclo da graduação e presença de disciplina de saúde mental. Isso porque os alunos do primeiro ciclo obtiveram maiores atitudes estigmatizantes do que os do último ciclo. Um estudo realizado com 5 cursos da área da saúde de uma instituição situada em Portugal também constatou essa diferença, corroborando com o presente estudo (Querido, Tomás, \& Carvalho, 2016).

Essa distinção também é reafirmada em outra pesquisa que encontrou níveis de estigma relativamente maiores nos alunos ingressantes, principalmente no quesito visão minoritária. Os autores atribuíram esse resultado à precariedade de conhecimentos específicos acerca da temática e à propagação de crenças e valores culturais ao longo das gerações, o que faz perpetuar o estigma frente às pessoas com transtorno mental e a precariedade de conhecimentos específicos acerca da temática (Mendes, Pereira, \& Scattolin, 2019), sendo que estes conhecimentos podem ser discutidos no ensino infantil, fundamental e médio, para que haja uma maior disseminação desse assunto, contribuindo positivamente na redução do estigma.

Da mesma forma, esta pesquisa evidencia que a presença da disciplina de saúde mental durante a graduação contribui na redução do estigma. Esse resultado é corroborado com outra pesquisa que também demonstrou que a passagem pela disciplina de saúde mental foi responsável por tornar a opinião dos estudante mais positiva, principalmente na categoria de etiologia de esforço mental (Ferreira, \& Carvalho, 2020).

Justifica-se essa correlação pela equivalência diretamente proporcional existente entre os conhecimentos em saúde mental e o nível de estigma. Isso acontece porque a existencia de conhecimentos contribui com o processamento adequado das informações que são disseminadas na população, o que finda por alteral o nível do estigma de tal forma que muito conhecimento 
irá reduzí-lo e, ao contrário, pouco conhecimento irá acentua-lo (Barrantes, Javier, Violante, Graca, \& Amorim, 2017).

Nesta pesquisa, os estudantes que têm contato com pessoas com transtorno mental possuem opiniões mais positivas na maioria dos domínios da escala utilizada. A eficiência desse contato é atingida por permitir um aumento na empatia, além de restringir atitudes mais negativas e pouco benevolentes (Corrigan et al., 2012; Poreddi et al., 2017; Yuksel, Yildiz, \& Coskun, 2019).

Logo, a mitigação do estigma após o contato com pessoas com transtorno mental reflete a contribuição do contato precoce entre alunos e pessoas em sofrimento mental, pois causa a interrupção de pensamentos negativos diante dessa população e, quiçá, possibilita uma melhor assistência na futura profissão deles.

Diante do exposto, percebe-se que há muitos fatores que influenciam as opiniões dos discentes e, consequentemente, nota-se a importância de conhecer estratégias que reduzam os níveis de estigma desse público. Isso pode ser auferido por meio da educação e do aumento do contato com as pessoas em sofrimento psíquico, o que irá contribuir positivamente na prática de estágios dos graduandos e na futura carreira profissional, refletindo também na qualidade da assistência prestada nos serviços de saúde.

Nesse sentido, algumas intervenções podem ser empregadas com a finalidade de amenizar as opiniões negativas dos estudantes para com os indivíduos com transtorno mental, a mencionar projetos de sensibilização que visem a compreensão dos transtornos mentais, disciplinas especificas sobre o tema, envolvimento dos alunos em ações antiestigma na comunidade e em locais de estágio, divulgação de informações por meio da mídia, intervenções que promovam o contato com as pessoas em sofrimento mental, e criação e implementação de programas que lidem com esse público (Rocha et al., 2015; Querido et al., 2016; Barrantes et al., 2017).

\section{Conclusões}

Este estudo avaliou as opiniões dos estudantes universitários de oito cursos da área da saúde frente às pessoas com transtornos mentais, e constatou, de forma geral, a presença da estigmatização na maioria dos cursos, com prevalência de menor estigma entre os discentes de Medicina e maior estigma entre os do curso de Odontologia.

Referente às limitação do estudo, houve diferença na quantidade relativa de questionários coletados quando realizado uma comparação geral entre os cursos, isso é reflexo de recusas dos alunos para responder o questionário. Além disso, iniciouse durante o período de coleta de dados as medidas de prevenção devido à pandemia do novo coronavírus (SARS-CoV-2).

Outro fator limitante foi a impossibilidade de realizar um estudo longitudinal a fim de investigar a presença de opniões estigmatizantes nos discentes ao decorrer do curso, reaplicando os mesmos questionários em cada ano da graduação. Sendo assim, estudos futuros podem ser realizados para suprir essa lacuna.

Apesar disso, os resultados desse estudo contribuem para a visualização da presença de opniões negativas e estigmatizantes dentre os universitários da área de saúde, o que serve de subsídio para mudança da estrutura curricular e para a criação de vivências precoces em ambientes focados em prevenção, promoção e tratamento de questões associadas à saúde mental pelas coordenações dos cursos a fim de sensibilizar os discentes e promover a redução do estigma mental.

Vale ressaltar que a redução do estigma mental é de suma importância, visto que este é uma das principais barreiras no cuidado às pessoas com transtorno mental, e que os discentes da área da saúde irão lidar com esse público em alguns momentos da graduação e, quiçá, durante a futura carreira profissional. Sendo assim, é mister que haja essa amenização para que se oferte cuidados de qualidade e possibilite a reinserção social desses indivíduos.

Em síntese do que foi exposto, há várias formas de atingir esse resultado, sendo que a mais importante é a associação de conteúdos teóricos com a prática, que irá disponibilizar conhecimentos acerca da saúde mental e possibilitar o contato com as pessoas portadoras de transtornos mental, respectivamente. Isso resultará em conscientização da realidade e desmitificação 
das crenças perpetuadas através das gerações, permitindo uma melhor convivência questões relacionadas à saúde mental.

\section{Referências}

Araújo, L. E. D., Sousa, F. D. A. S. D., Ribeiro, M. A. D. F. M., Santos, A. S. D., \& Medeiros, P. D. C. (2008). Análise estatística de chuvas intensas na bacia hidrográfica do rio Paraíba. Revista Brasileira de Meteorologia, 23(2), 162-169.

Arboleda-Flórez J., \& Stuart H. (2012). From sin to science: Fighting the stigmatization of mental illnesses. Revue Canadienne De Psychiatrie, 57(8), 457-463.

Aruna G., Mittal S., Yadiyal M.B., Acharya C., Acharya S., \& Uppulari C. (2016). Perception, knowledge, and attitude toward mental disorders and psychiatry among medical undergraduates in Karnataka: A cross-sectional study. Indian Journal of Psychiatry, 58(1), 70-6, 2016.

Barbosa T.R. (2010). Estigma face à doença mental por parte de futuros profissionais de saúde mental. Dissertação de mestrado, Faculdade de Psicologia e de Ciências da Educação da Universidade do Porto, Portugal.

Barrantes F.J., Javier F., Violante C., Graca L., \& Amorim I. (2017). Programa de Luta conta o Estigma: resultados obtidos na formação nos profissionais da Saúde Mental. Revista Portuguesa de Enfermagem de Saúde Mental [Internet], 5 (SPE5), 19-24.

Brasil (2012). Resolução CNS Nº 466/12: Dispõe sobre pesquisas com seres humanos. Brasília: Ministério da Saúde, Conselho Nacional de Saúde.

Cândido M.R., Oliveira E., Monteiro C., \& Costa J.R., Benício S.R., Costa F.L. (2012). Conceitos e preconceitos sobre transtornos mentais: um debate necessário. Revista Eletrônica Saúde Mental Álcool Drog [Internet], 8(3), 110-117.

Canfield A.A. (1951). The "Sten” Scale-A Modified C-Scale. Educ Psychol Meas, 11(2), 295-7.

Chang S., Ong H.L., Seow E., Chua B.Y., Abdin E., Samari E., Teh W.L., Chong S.A., \& Subramaniam M. (2017). Stigma towards mental illness among medical and nursing students in Singapore: a cross-sectional study. BMJ Open [Internet], 7(12).

Corrigan P.W., Morris S.B., Michaels P.J., Rafacz J.D., \& Rüsch N. (2012). Challenging the public stigma of mental illness: A meta-analysis of outcome studies Psychiatric Services [Internet], 63, 963-973.

Delevati, D. M., \& Palazzo, L. D. S. (2008). Atitudes de empresários do Sul do Brasil em relação aos portadores de doenças mentais. Jornal Brasileiro de Psiquiatria, 57(4), 240-246.

Ferreira F.N., Fernandino D.C., Souza G.R.M., Ibrahim T.F., Fukino A.S.L., Araújo N.C., \& Vidal C.E.L. (2015). Avaliação das atitudes de estudantes da área da saúde em relação a pacientes esquizofrênicos. Revista Brasileira de Educação Médica [Internet], 39(4), 542-557.

Ferreira M.S., \& Carvalho M. (2017). Estigma associado ao transtorno mental: uma breve reflexão sobre suas consequências. Revista Interdisciplinar de Estudos em Saúde, 6(2), 192-201.

Ferreira M.S., \& Carvalho M.C. (2020). Educação para o enfrentamento do estigma: uma intervenção educacional com alunos de enfermagem. Revista Portuguesa de Enfermagem de Saúde Mental, (23), 15-22.

Fresán A., Robles-García R., Martínez-López N., Tovilla-Zárate C.A., \& Madrigal E. (2018). Stigma and perceived aggression towards schizophrenia in female students of medicine and psychology. Salud Mental [Internet], 41(5).

Jorge M.R. (2013). Concepções populares e estigma relacionados às doenças mentais. Nova Perspectiva Sistêmica, 22(46), 8-19.

Lim H.J., Moxham L., Patterson C., Perlman D., Lopez V., \& Goh Y.S. (2019). Students' mental health clinical placements, clinical confidence and stigma surrounding mental illness: A correlational study. Nurse Education Today, 84.

Mathew C.P. (2017). Impact of internalized stigma on life satisfaction of persons with mental illness. Indian Journal of Applied Research, 7(10), 574-577.

Mendes A.O., Pereira C.M., \& Scattolin F.A. (2019). Opiniões de estudantes de um curso de graduação em Enfermagem sobre a doença mental. Revista da Faculdade de Ciências Médicas de Sorocaba, 21(2): 69-76.

McKight, P. E., \& Najab, J. (2010). Kruskal-wallis test. The corsini encyclopedia of psychology, 1-1.

Ordan R., Shor R., Liebergall-Wischnitzer M., Noble L., \& Noble A. (2018). Nurses' professional stigma and attitudes towards postpartum women with severe mental illness. Journal of Clinical Nursing [Internet], 27(7-8), 1543-1551.

Organização Mundial da Saúde (2005). Livro de recursos da OMS sobre saúde mental, direitos humanos e legislação: Cuidar, sim - excluir, não (p. 164). Genebra: OMS.

Perme, M. P., \& Manevski, D. (2019). Confidence intervals for the Mann-Whitney test. Statistical methods in medical research, 28(12), 3755-3768.

Poreddi V., Thimmaiah R., \& Math B.S. (2017). Medical and nursing students' attitudes toward mental illness: An Indian perspective. Investigación y Educación en Enfermería [Internet], 34(1), 86-94.

Querido A., Tomás C., \& Carvalho D. (2016). O Estigma face à doença mental nos estudantes de saúde. Revista Portuguesa de Enfermagem de Saúde Mental [Internet], (SPE3), 67-72.

Resolução n 22/2015/CONEPE. Aprova alterações no Projeto Pedagógico do Curso de Graduação em Fonoaudiologia - Bacharelado do Campus Universitário Prof. Antônio Garcia Filho. Departamento de Fonoaudiologia. Sergipe: Autores. 
Research, Society and Development, v. 10, n. 14, e269101422134, 2021

(CC BY 4.0) | ISSN 2525-3409 | DOI: http://dx.doi.org/10.33448/rsd-v10i14.22134

Resolução no 25/2017/CONEPE. Aprova alterações no projeto pedagógico do curso de graduação em Odontologia Bacharelado do Campus Universitário Prof. Antônio Garcia Filho e dá outras providências. Departamento de Odontologia. Sergipe: Autores.

Rocha F.L., Hara C., \& Paprocki J. (2015). Doença mental e estigma. Revista Médica de Minas Gerais, 25(4), 590-596.

Rodrigues C.R. (1983). Atitudes frente a doença mental: Estudo de uma amostra de profissionais da saúde. Tese de doutorado, Faculdade de Medicina de Ribeirão Preto, SP, Brasil.

Sari S.P., \& Yuliastuti E. (2018). Investigation of attitudes toward mental illness among nursing students in Indonesia. International Journal of Nursing Sciences [Internet], 5(4), 414-418.

Silva J.J., \& Carvalho J. (2017). Pontes para a inclusão: O combate ao estigma na doença mental. Revista Pró-UniverSUS, 8(2), 47-51.

Silva, M. J., Vilaça, S., Martins, C. A., \& Oliveira, C. (2015). A escrita de casos em Problem Based Learning: uma experiência no ensino de enfermagem. Revista Iberoamericana de Educación e Investigación en Enfermería, 5(2), 58-64.

Thornicroft G., Mehta N., Clement S., Evans-Lacko S., Doherty M., Rose P.D., Koschorke M., Shidhaye R., O’Reilly C., \& Henderson C. (2016). Evidence for effective interventions to reduce mental-health-related stigma and discrimination. The Lancet, 387, 1123-1132.

Vistore A., Ribeiro W., Ziebold C., Asevedo E., Evans-Lacko S., Keeley J.W., Gonçalves D.A., Palacios N.G., \& Mari J.J. (2018). Clinical decisions and stigmatizing attitudes towards mental health problems in primary care physicians from Latin American countries. Plos One [Internet],13(11), 2-11.

Weisstein, E. W. (2004). Bonferroni correction. https://mathworld.wolfram.com/.

Yuksel G., Yildiz M., \& Coskun B. Medical faculty students' beliefs toward mental illness and the impact of visiting a community mental health center on these beliefs. Dusunen Adam The Journal of Psychiatry an Neurological Sciences, 2(32), 152-160. 